\title{
Cómo cambiar comportamientos durante una pandemia: El uso de nudges para enfrentar la COVID-19
}

\section{How to change behaviours during a pandemic: The use of nudges to confront COVID-19}

\author{
Hugo Cuello Díaz \\ MPP Hertie School of Governance (Alemania) \\ h.cuello@outlook.com
}

\begin{abstract}
NOTA BIOGRÁFICA
Licenciado en Ciencias Políticas y de la Administración por la Universidad Complutense de Madrid (UCM) y Máster en Políticas Públicas por la Hertie School of Governance de Berlín. Actualmente es investigador en el Innovation Growth Lab (IGL) de la fundación británica de innovación Nesta, donde asesora a una docena de gobiernos de todo el mundo para diseñar experimentos que mejoren sus políticas de innovación y crecimiento económico. Ha trabajado para la Agencia de Cooperación Alemana (GIZ) en Centroamérica y en la Unidad de Evaluación de políticas públicas del Gobierno de Costa Rica. Además, ha asesorado a empresas y centros de investigación en Europa y el Sudeste Asiático.
\end{abstract}

\section{RESUMEN}

El presente estudio analiza la situación actual del uso de nudges para enfrentar la pandemia de la COVID-19. Se presentan las justificaciones teóricas y prácticas para el uso de los nudges durante una pandemia, así como ejemplos concretos, estudio de patrones y limitaciones del enfoque. Los nudges pueden considerarse como una herramienta complementaria a las restricciones de comportamiento que se han presentado en la mayoría de los países. Su enfoque puede ser efectivo a la hora de lograr los cambios de comportamiento necesarios para reducir el número de contagios, como incentivar a la gente a quedare en casa o lavarse las manos de forma regular.

\section{PALABRAS CLAVE}

Nudges; Economía del Comportamiento; COVID-19.

\begin{abstract}
The present study explores the current situation of the use of nudges to face the COVID-19 pandemic. The paper presents theoretical and practical explanations for the use of nudges during a pandemic, as well as concrete examples, patterns and limitations of the approach. Nudges can be considered as a complementary behavioural tool to the restrictions that have been imposed in several countries. This approach can be effective in bringing the behavioural changes needed to reduce the number of infections, such as encouraging people to stay home or washing their hands on a regular basis.
\end{abstract}

\section{KEYWORDS}

Nudges; Behavioural Economics; COVID-19. 
GAPP. Nueva Época - N. ${ }^{25}$, marzo 2021 - ISSN: 1989-8991 - DOI: https://doi.org/10.24965/gapp.i25.10873 - [Págs. 73-86] Número monográfico - Los nudges y el diseño conductual de políticas públicas

Cómo cambiar comportamientos durante una pandemia: El uso de nudges para enfrentar la COVID-19.

Hugo Cuello Díaz

\section{SUMARIO}

1. INTRODUCCIÓN: LA PANDEMIA DE LA COVID-19 Y LOS NUEVOS COMPORTAMIENTOS DE LA POBLACIÓN. 2. ¿POR QUÉ NECESITAMOS NUDGES? LIMITACIONES DE LAS RESTRICCIONES PARA INCENTIVAR COMPORTAMIENTOS. 3. COMPORTAMIENTOS VIRALES. SESGOS Y MECANISMOS PSICOLÓGICOS DE COMPORTAMIENTO DURANTE UNA PANDEMIA. 4. EJEMPLOS DE NUDGES PARA ENFRENTAR LA PANDEMIA DE LA COVID-19. 5. PATRONES IDENTIFICADOS Y ESTRATEGIAS DE ÉXITO PARA DISEÑAR NUDGES DURANTE LA COVID-19. 6. CONCLUSIÓN: IDEAS Y LIMITACIONES EN EL USO DE NUDGES PARA ENFRENTAR LA COVID-19. REFERENCIAS BIBLIOGRÁFICAS.

\section{INTRODUCCIÓN: LA PANDEMIA DE LA COVID-19 Y LOS NUEVOS COMPORTAMIENTOS DE LA POBLACIÓN}

En diciembre de 2019, las autoridades chinas notificaron 27 casos de neumonía de origen desconocido. Los casos fueron vinculados a un nuevo tipo de coronavirus SARS-CoV-2 que causa la enfermedad por coronavirus COVID-19. Tanto este nuevo virus como la enfermedad que provoca eran desconocidos antes de que estallara el brote en Wuhan (China) a finales de 2019.

Actualmente la COVID-19 es una pandemia que ha alterado la vida de la gran mayoría de la población mundial. Más de seis meses desde los primeros casos oficiales en China, los contagiados se cuentan por millones y los muertos oficiales entre enero y julio de 2019 son más de 500.000 . La expansión del virus los meses posteriores afectó con especial gravedad a Europa y Estados Unidos, que impusieron duras medidas para controlar el virus. Meses después, se extendió también por zonas del Sur de Asia y Latinoamérica.

En España, los datos de la segunda ronda del estudio ENE-COVID dejan entrever el porcentaje aproximado de población que se contagió durante los primeros meses de la pandemia. Según estos estudios sero-epidemiológicos realizados por el Ministerio de Sanidad, la prevalencia estimada de anticuerpos IgG frente al SARS-Cov2 en la población española es de un 5,21\% (Ministerio de Sanidad, 2020). Es decir, aproximadamente 2.5 millones de españoles fueron contagiados durante los primeros meses de la pandemia. Con fecha de mediado de julio de 2020, en España las cifras oficiales saldaban una cuenta de más de 28.000 fallecidos. Sin embargo, para el mismo periodo otros estudios ponen la cifra de fallecidos en España más cercana a las 45.000 personas (Romero, 2020).

El virus en España fue contenido gracias a una serie de medidas estrictas que implicaron el confinamiento de toda la población española durante casi tres meses. De hecho, según una investigación de la Universidad de Oxford, España fue uno de los países que aprobó las restricciones más duras tras el estallido de la pandemia (Hale et al., 2020). Sin embargo, cuando algunas de las medidas más estrictas fueron levantadas y se comenzó una nueva fase conocida como la «nueva normalidad», el número de contagios fue creciendo de forma exponencial de nuevo. En el momento de escribir estas líneas, España se ha convertido en el país europeo con la mayor tasa de contagios (RTVE.es, 2020).

La situación va cambiando cada día y es muy complicado hacer predicciones y análisis a largo plazo de los efectos del virus, ya que depende enormemente de cómo evolucione en los próximos meses y de la rapidez con la que lleguen las vacunas. Sin embargo, podemos empezar a estudiar las diferentes respuestas que los gobiernos de todo el mundo han tenido durante los peores meses de pandemia y cómo el comportamiento de los ciudadanos ha influido en esa evolución.

La respuesta a la COVID-19 ha sido muy diferente entre países, y dará pie a años de estudio en ciencias sociales sobre las interconexiones entre la credibilidad y confianza en las instituciones, la respuesta ciudadana, las capacidades de respuesta pública y la toma de decisiones.

En lo referente a la ciudadanía, la mayoría de los gobiernos europeos concentró su actividad en imponer medidas restrictivas para frenar la expansión del virus. Medidas de confinamiento que han reducido las libertades individuales, como libertad de movimiento y de reunión.

Las medidas de confinamiento estricto como las vividas en España durante el estado de alarma, si bien fueron efectivas para bajar la curva de contagios en su peor momento, no son un modelo que pueda ser extendido ad infinitum. El confinamiento tiene un efecto muy pernicioso en la economía de un país. Además, tiene muchos efectos negativos en la salud mental de las personas y otros indicados sociales (Brooks et al., 2020).

La única manera que tenemos de controlar el virus mientras no haya vacuna, es por medio del cambio de comportamiento de la población. La naturaleza de la COVID-19 lleva a considerar la importancia que pue- 
GAPP. Nueva Época - N. ${ }^{\circ}$ 25, marzo 2021 - ISSN: 1989-8991 - DOI: https://doi.org/10.24965/gapp.i25.10873 - [Págs. 73-86] Número monográfico - Los nudges y el diseño conductual de políticas públicas

Cómo cambiar comportamientos durante una pandemia: El uso de nudges para enfrentar la COVID-19.

Hugo Cuello Díaz

dan tener medidas efectivas basadas en el comportamiento. Hasta que el acceso a una vacuna se extienda de forma que una parte significativa de la población esté inmunizada, se necesitan medidas de contención que los epidemiólogos denominan «intervenciones no farmacéuticas» (Wang et al. 2020), entre las que incluyen distanciamiento social, lavado de manos o uso de mascarillas. En otras palabras, cambios de comportamiento. La pregunta que muchos gobiernos intentan responder ahora es, ¿cómo conseguir y mantener esos cambios de comportamiento de forma efectiva?

El cumplimiento de estas medidas tiene un efecto directo en la salud pública, además de impacto en las oportunidades para salvar miles de vidas. Cuando un comportamiento se multiplica por toda la población, un aparente pequeño cambio tiene el poder de acelerar o frenar una pandemia. Esta situación pone el estudio y análisis de los comportamientos en el centro de las decisiones públicas.

Existen algunos comportamientos que se han adoptado durante la pandemia, entre los que destacan algunos ${ }^{1}$ :

- Quedarse en casa.

- Mantener la distancia social al menos 2 metros con otras personas.

- Llevar mascarilla.

- Lavarse las manos de forma frecuente por al menos 20 segundos.

- Desinfectar superficies.

- Toser o estornudar hacia el codo.

Algunos de estos comportamientos como lavarse las manos de forma frecuente se desarrollan en base a hábitos ya previamente existentes. Pero otros comportamientos son completamente nuevos, como mantener la distancia social. Esto crea dificultades añadidas para los ciudadanos a la hora de cumplir algunas de estas medidas y nos lleva a la pregunta esencial que se lleva intentando responder en diseño de políticas públicas desde hace décadas: ¿Cómo se crean y consolidan nuevos hábitos en el comportamiento de la población?

Este artículo intenta responder a algunas preguntas y exponer la justificación, implementación y limitaciones del uso de nudges para luchar contra el virus. El tema es altamente relevante, ya que como explica la Organización Mundial de la Salud (OMS): «la perspectiva del comportamiento es valiosa para informar la planificación de medidas adecuadas para responder a la pandemia» (OMS, 2020b). De hecho, la OMS ha creado una herramienta online usando la ciencia del comportamiento y está contratando expertos en el terreno (OMS, 2020a) $)^{2}$.

La experiencia de la OMS durante años, enfrentándose a diferentes pandemias, les ha expuesto la importancia de contener epidemias cambiando comportamientos. En estos casos, las medidas son mucho más cognitivas que médicas. Los nudges (o impulsos, en castellano) son estímulos que conscientemente se sitúan en el entorno o en los mensajes que reciben los ciudadanos para guiar sus decisiones. Muchos de ellos derivan de la investigación experimental y están orientados a mejorar la educación o la salud de la población (Hummer \& Meadche, 2019). Son herramientas necesarias para consolidar los hábitos de comportamiento, especialmente tras la primera ola de contagios cuando los ciudadanos dejaron de sentir el riesgo inmediato y existe una sobrecarga psicológica por el shock de la situación.

En la siguiente sección se presentan las razones por las cuáles los nudges pueden ser una herramienta útil en ciertos contextos, complementarias a las actuales restricciones que no siempre han conseguido los objetivos esperados. A continuación, la sección 3 observa en más detalle la justificación teórica y mecanismos psicológicos que llevan al cambio de comportamiento en una pandemia y cómo se pueden usar para diseñar nudges. La sección 4 presenta una serie de nudges desarrollados durante los primeros meses de la pandemia en diversos países, específicamente para mantener la distancia social y lavarse las manos de forma regular. A continuación, la sección 5 recopila las lecciones de los nudges existentes para informar el posible diseño de futuras intervenciones públicas. Finalmente, la sección 6 presenta las conclusiones y limitaciones del uso de nudges para enfrentar la pandemia de la COVID-19.

1 La lista de los principales comportamientos recomendados se puede encontrar en la web de la Organización Mundial de Salud: "Coronavirus disease (COVID-19) advice for the public". Disponible en: https://www.who.int/emergencies/diseases/novel-coronavirus-2019/advice-for-public.

2 El proceso de selección de expertos de la OMS se puede encontrar en "Call for experts on behavioural insights and sciences for public health" del 3 de Marzo 2020. Disponible en: https://www.who.int/news-room/articles-detail/call-for-experts-on-behaviouralinsights-and-sciences-for-public-health. 
GAPP. Nueva Época - N. 25, marzo 2021 - ISSN: 1989-8991 - DOI: https://doi.org/10.24965/gapp.i25.10873 - [Págs. 73-86] Número monográfico - Los nudges y el diseño conductual de políticas públicas

Cómo cambiar comportamientos durante una pandemia: El uso de nudges para enfrentar la COVID-19.

Hugo Cuello Díaz

\section{2. ¿POR QUÉ NECESITAMOS NUDGES? LIMITACIONES DE LAS RESTRICCIONES PARA INCENTIVAR COMPORTAMIENTOS}

Introducir nuevos comportamientos en la población ha sido históricamente un reto complejo para los decisores públicos. La mayoría de las medidas que han buscado incentivar comportamientos positivos se han basado tradicionalmente en ofrecer incentivos positivos, como pequeñas recompensas económicas, 0 negativas como multas, sanciones o prohibiciones (Thaler \& Sunstein, 2009).

En España, las medidas para incentivar el cambio de comportamiento frente a la COVID-19 han seguido esta línea. Durante los últimos meses, se ha creado un nuevo cuerpo normativo desarrollando sanciones por algunas actividades que pueden aumentar el riesgo de contagio, por ejemplo, prohibiendo reuniones de más de 10 personas. Cada comunidad autónoma ha decidido cuál es el castigo proporcional por algunos de estos comportamientos, que pueden llegar hasta los 3.000 euros por ir a una fiesta sin mascarilla (Bohórquez, 2020). Estas medidas sancionadoras habrían sido incomprensibles y políticamente inviables si no hubiéramos sufrido la peor catástrofe sanitaria en más de 100 años.

El problema es que muchas de estas medidas no son negativas de por sí, ni parecerían sancionables en condiciones normales. Es más, son parte natural del comportamiento básico de los individuos, que además mejoran enormemente su situación psicológica en un momento donde más se necesita. Reunirse con amigos, socializar, es un elemento esencial del ser humano y preséntalos de forma permanente como un comportamiento punitivo puede crear efectos perversos (Umberson \& Montez, 2010).

En general, los españoles han respondido adecuadamente y cumplieron con las peticiones de las autoridades sanitarias. La mayoría de los españoles cumplieron con las estrictas medidas impuestas durante los peores meses de la pandemia. Esto se observa en varios indicadores, como lo datos de movilidad presentados por Google, que mostraron que el $90 \%$ de los españoles no se movió de su zona durante el confinamiento, un porcentaje más alto que otros países de su entorno (Google, n. d.).

De igual forma, el uso de mascarillas se ha generalizado antes incluso de imponer su obligatoriedad en la mayoría de las comunidades autónomas. Según datos del centro demoscópico YouGov, España fue el país que tuvo un crecimiento exponencial más rápido en el uso de mascarillas y el país europeo cuyos ciudadanos afirmaban estar más dispuestos a usarlas (Smith, 2020).

Sin embargo, el nuevo aumento de los contagios y el enorme impacto que han tenido los rebrotes ha llevado a considerar si este enfoque es el más adecuado. Al no tener evidencia clara de qué está causando la mayoría de los rebrotes, las autoridades están imponiendo restricciones y medidas punitivas sobre la población. Sin embargo, otros países con medidas mucho más laxas en el cumplimiento de estos comportamientos han tenido rebrotes más controlados y gestionables que España, lo que hace indicar que no solo es una cuestión de imponer de forma estricta la obligatoriedad del uso de mascarillas en todos los espacios y la distancia social. La comunidad médica está de acuerdo en que estos comportamientos son esenciales, pero también hay otros elementos sistémicos. De hecho, un buen número de expertos médicos en España concuerdan en que las capacidades de rastreo y de respuesta en la atención primaria, así como recursos sanitarios o ventilación adecuada son las principales causas de por qué en España hemos sido incapaces de controlar los rebrotes (Güell, 2020). Sorprendentemente, la mayoría de ellas no tienen vinculación con el comportamiento ciudadano, sino que su respuesta es institucional y política.

De hecho, a nivel internacional hay expertos médicos pidiendo un cambio de enfoque generalizado. Julia Marcus, epidemióloga y profesora de la Harvard Medical School argumenta que es normal que, aunque las reuniones sociales sin tomar las medidas nos puedan producir indignación, castigarlas por ley llevaría aumentar la injusticia y no reduciría el número de infecciones (Marcus, 2020).

Estos expertos argumentan que existen casos de criminalización de comportamientos para evitar la propagación de un virus que no han funcionado. Por ejemplo, exponer a alguien al VIH -incluido con actividades sexuales que no suponen ningún riesgo- es un crimen en países como Estados Unidos o Canadá (Hasenbush et al., 2015). Y según la mayoría de expertos médicos, estas leyes han sido un completo fracaso, especialmente para proteger a comunidades marginadas y vulnerables (The Lancet HIV, 2018).

Como con el VIH, la COVID-19 no actúa igual en todas las clases sociales (Ford et al., 2020). Por tanto, la criminalización de estas actividades afectará más a los grupos vulnerables. En Nueva York, la policía demanda cumplir las medidas de distanciamiento social y casi todos los arrestos son entre la comunidad negra y latina. Incluyendo casos con presencia de violencia policial (Southall, 2020). En España y otros países europeos, algunos discursos públicos fueron en la línea de señalamiento de algunas poblaciones, como 
GAPP. Nueva Época - N.o 25, marzo 2021 - ISSN: 1989-8991 - DOI: https://doi.org/10.24965/gapp.i25.10873 - [Págs. 73-86] Número monográfico - Los nudges y el diseño conductual de políticas públicas

Cómo cambiar comportamientos durante una pandemia: El uso de nudges para enfrentar la COVID-19.

Hugo Cuello Díaz

jóvenes, inmigrantes o trabajadores del campo ${ }^{3}$. Existen casos muy destacados, como el pueblo en la Isla de Gran Canaria que montó barricadas para impedir la entrada de inmigrantes por riesgo de contagio, aunque todos habían dado negativo en los test (Álamo, 2020).

Alternativamente, en algunas regiones como el Distrito de British Columbia en Canadá, se ha vinculado su bajo nivel de contagios con el enfoque llevado por las autoridades y su intención de no involucrar a la policía más de lo necesario (Obliko, 2020). La responsable de salud de la provincia canadiense, en base a su amplia experiencia con el Ébola, argumenta que la mejor forma de promover comportamientos positivos durante emergencias sanitarias es por medio de una comunicación clara y empática, no con medidas punitivas (Porter, 2020).

Bajo estas circunstancias, sería útil considerar un enfoque complementario al de las restricciones que permita en cierta medida relajar algunas de estas medidas cuando sea posible, pero sin que eso conlleve un aumento automático de las infecciones. Para ello, los nudges pueden ser un instrumento adicional en la caja de herramientas de los decisores públicos.

\section{COMPORTAMIENTOS VIRALES. SESGOS Y MECANISMOS PSICOLÓGICOS DE COMPORTAMIENTO DURANTE UNA PANDEMIA}

Cuando se diseñan soluciones públicas, es fácil reconocer barreras físicas y económicas que impiden su cumplimiento, pero los decisores se olvidan de las barreras psicológicas de forma regular. En general, la población tiende a pensar las elecciones sobre su comportamiento, especialmente en momentos de pandemia mundial, están basadas puramente en decisiones racionales, alimentadas en la mejor información posible. Pero la evidencia de la ciencia del comportamiento y psicología social demuestra que este no suele ser el caso (Gigerenzer \& Gaissmaier, 2011). Para diseñar un nudge que sea efectivo, antes hay que analizar los comportamientos deseables y entender cuáles son las barreras que están impidiendo su cumplimiento.

Un elemento central en el estudio de barreras psicológicas durante una pandemia es el tipo de comunicación y estrategias llevadas a cabo para informar de forma efectiva a la población. Expertos en pandemias han investigado este elemento, destacando la dificultad que implica que la comunidad comprenda estos mensajes basados en la salud pública. No solo centrados en protegerse a uno mismo, sino en proteger a los demás. Por ello, se han creado herramientas como la Communication for Behavioural Impact (COMBI), una herramienta para mejorar la comunicación basada en nudges (OMS, 2012).

Encontrar el nudge correcto no consiste en probar todas las estrategias psicológicas a la vez ni en copiar los que funcionaron en otros espacios y replicarlos sin más. Se necesita una revisión previa detallada del contexto y de la situación en el que se quiere aplicar, entendiendo las barreras existentes en ese espacio o grupo poblacional (Martela \& Riekki, 2018). Los expertos en economía del comportamiento y diseño de nudges insisten que la primera pregunta a responder es qué tipo de sesgos cognitivos existen y dificultan la adopción de comportamientos positivos en esa localización geográfica o sector de la población (Abendroth-Dias, 2020).

Para entender cuáles son las barreras que están confrontando con esos comportamientos, existen modelos como el modelo COM-B de la Univerity Collegue of London que facilita dicho análisis (West \& Michie, 2020). Según el modelo, para que la población introduzca nuevos comportamientos se necesitan tres condiciones: Capacidad, Oportunidad y Motivación. Esto significa que, por ejemplo, la población debería conocer cómo lavarse las manos de forma correcta, tener agua y jabón cerca, y entender la importancia de dicha acción para luchar contra la COVID-19. Observando dicho modelo, podemos investigar qué barreras existen para comportamientos específicos en un grupo particular de población.

Existen, sin embargo, elementos que añaden dificultad al análisis. La teoría psicológica expone que la mayoría de los seres humanos tiene tres necesidades psicológicas básicas, que se pueden encontrar en la mayoría de los contextos, países y poblaciones. Son la necesidad de autonomía, de competencia y de relacionalidad o parentesco (Ryan \& Deci, 2017). La autonomía implica la capacidad de tomar decisiones sobre las propias necesidades. La competencia implica la interacción con el entorno y reconocer la posibilidad de marcar la diferencia. Y la relacionalidad es la necesidad de conexión con otras personas. Todos los individuos, independientemente de su personalidad y situación, tienen estas necesidades psicológicas (Michie et al., 2011).

3 Para conocer el caso de Italia como ejemplo, véase el artículo del New York Times, "As Coronavirus Reappears in Italy, Migrants Become a Target for Politicians". URL: https://www.nytimes.com/2020/08/28/world/europe/coronavirus-italy-migrants.html. 
GAPP. Nueva Época - N. 25, marzo 2021 - ISSN: 1989-8991 - DOI: https://doi.org/10.24965/gapp.i25.10873 - [Págs. 73-86] Número monográfico - Los nudges y el diseño conductual de políticas públicas

Cómo cambiar comportamientos durante una pandemia: El uso de nudges para enfrentar la COVID-19.

Hugo Cuello Díaz

Parte de lo que hace tan complejo el cambio de comportamiento para enfrentar a la COVID-19 es que los cambios que se buscan afectan de una u otra manera a estas necesidades psicológicas. Un nudge efectivo necesitará ser diseñado para aumentar el sentimiento de autonomía, competencia y relacionalidad, mientras intenta cubrir las barreras del cambio de comportamiento. Además, hay que considerar las circunstancias antropológicas específicas de cada territorio, que pueden incluir en la motivación de los ciudadanos a cumplir con ciertas medidas.

Dada la naturaleza de la pandemia, que demanda intervenciones que motiven el cambio de comportamiento a nivel macro y entre varios sectores, se han presentado varios nudges y otras intervenciones en varios países que motiven a adoptar estos comportamientos, basados en normas sociales, deber cívico, empatía y confianza en la ciencia. La investigación ha sido rápida y se actualiza con mucha regularidad, de forma que los resultados hay que tomarlos con precaución. En la siguiente sección se presentan algunos ejemplos que se han desarrollado durante los primeros 6 meses de la pandemia.

\section{EJEMPLOS DE NUDGES PARA ENFRENTAR LA PANDEMIA DE LA COVID-19}

Algunas organizaciones y gobiernos locales y nacionales ya han comenzado a investigar la aplicación de nudges en sus regiones. Por ejemplo, el Behavioural Inisights Team (BIT) ha desarrollado aproximadamente 40 experimentos entre más de 80,000 personas probando diferentes nudges en temas claves como el lavado de manos, mascarillas y mantener la distancia social (Halerm \& Harper, 2020).

La comunicación durante una pandemia es clave y el uso de diferentes mensajes puede tener efectos muy diferentes. Desde las grandes campañas gubernamentales para incitar a la gente a quedarse en casa, hasta pequeños pósteres y folletos que incentiven a lavarse las manos de la forma correcta.

A simple vista, el uso de uno u otro mensaje puede parecer un asunto menor en momentos tan críticos como el actual. Sin embargo, la evidencia empírica demuestra que es una cuestión a tener en cuenta si queremos que los recursos públicos dedicados a luchar contra la pandemia sean efectivos y reduzcan los contagios al menor coste posible, tanto económico y psicológico.

La forma para evaluar la efectividad de los nudges se basa normalmente en experimentos rigurosos, también conocidos como ensayos controlados aleatorios (Randomised Controlled Trial en inglés, o RCT). Los RCTs son considerados como el «estándar de oro» en la evaluación de impacto de políticas públicas. Ofrecen la posibilidad de comparar una intervención o un mensaje específico a un grupo de población, frente a un grupo de control elegido aleatoriamente que no recibe este mensaje. Con un experimento bien diseñado y con suficiente poder estadístico (una muestra grande y representativa), se asegura que no hay variables externas influyendo en el resultado. Por tanto, cualquier diferencia entre el grupo de tratamiento (que recibe el nudge) y el grupo de control es exclusivamente causada por el impacto del nudge.

Para probar los nudges de forma rápida y sencilla, muchos gobiernos se han sostenido en experimentos online, conocidos como «A/B test», donde una muestra poblacional se divide en dos o más grupos aleatoriamente, y cada uno recibe un mensaje diferente vía email, SMS, redes sociales o carta postal.

Durante los últimos meses, se han elaborado experimentos sobre nudges en diferentes materias. Una selección de los nudges más relevantes se presentan a continuación.

\subsection{Nudges para promover el lavado de manos}

Lavarse las manos es un hábito sanitario que ya se recomendaba antes de que la pandemia hiciera estragos en la población. Sin embargo, muchos estudios han demostrado la dificultad de hacerlo de forma efectiva y consistente (De Buck et al., 2017).

En 2008, un estudio en Reino Unido demostró que el $28 \%$ de personas que se encontraban de camino al trabajo tenían bacterias fecales en sus manos (Judah et al., 2010). Este ha sido un problema generalizado, que se ha encontrado hasta en profesionales sanitarios. Un $70 \%$ de las infecciones en hospitales, que matan casi a 100.000 personas al año en Estados Unidos, podrían evitarse si los profesionales sanitarios siguieran los protocolos sanitarios recomendados, incluyendo el lavado de manos (Umscheid et al., 2011).

Se han probado diversas intervenciones para motivar a la gente a lavarse las manos de forma consistente. (Vindigni et al., 2011). Muchas han resultado difíciles de evaluar, ya que la gente tiende a reportar mayor lavado de manos del que hace realmente y muchas veces es complicado mantener esos comportamientos en el tiempo (Manun'Ebo et al., 1997). 
GAPP. Nueva Época - N. ${ }^{25}$, marzo 2021 - ISSN: 1989-8991 - DOI: https://doi.org/10.24965/gapp.i25.10873 - [Págs. 73-86] Número monográfico - Los nudges y el diseño conductual de políticas públicas

Cómo cambiar comportamientos durante una pandemia: El uso de nudges para enfrentar la COVID-19.

Hugo Cuello Díaz

Este tipo de comportamientos son difíciles de explicar desde una perspectiva puramente utilitarista. Si fuera un problema exclusivamente de acceso físico al recurso, no tendría por qué estar relacionado con una barrera cognitiva. De hecho, la evidencia demuestra que ofrecer más puestos de lavado tiene un efecto positivo en el aumento de esta práctica (Pickering et al. 2018). Sin embargo, no es suficiente para extender su uso generalizado, ya que existen razones cognitivas más complejas que limitan esta práctica.

Se han considerado explicaciones teóricas basadas en economía del comportamiento sobre cuáles son las barreras cognitivas que nos dificultan hacer el lavado de manos de forma efectiva. En este caso, se cumplen las condiciones de capacidad, oportunidad y motivación necesarias para motivar el cambio de comportamiento con el uso de nudges.

Existe evidencia demostrando que las limitaciones de atención y esfuerzo naturales a cualquier ser humano afectan a este tipo de medidas que son aparentemente sencillas. Fatiga, agotamiento psicológico, procrastinación y otros sesgos afectan a nuestra habilidad de cumplir con el lavado de manos en condiciones normales (Dai et al., 2015). Es un claro ejemplo de lo que se conoce como la brecha entre intención y cumplimiento: Aunque tengamos la intención de lavarnos las manos, en el contexto inmediato no lo hacemos (Sheeran \& Webb, 2016). En época de pandemia, puede ser que la atención de los ciudadanos esté puesta en otras medidas más visibles como la mascarilla, lo que hace que accidentalmente le quiten importancia al lavado de manos. Con esta información, la ciencia del comportamiento ofrece una sólida base para diseñar nudges que confronten con las barreras reales de la población a la hora de lavarse las manos.

Un nudge en forma de recordatorios en el momento adecuado pueden ayudar a romper con esa brecha. En Reino Unido se experimentó con el uso de diferentes nudges en dispensadores de jabón en estaciones de tren. El más efectivo, basado en el uso de normas sociales y comportamiento socialmente aceptable, aumentó el uso de jabón más de un 10 \% (Judah et al., 2009). En este caso, el elemento de relacionalidad demostró ser particularmente importante, aunque el impacto de los nudges variaba enormemente por género.

Ya cuando la pandemia estaba presente, BIT estudió sobre la población británica el efecto de diferentes nudges que se llevaron a cabo en varios países. Se evaluaron 7 folletos utilizados por las autoridades de sus respectivos países, para identificar cuáles son los principios clave que ofrecían un mejor desempeño, tanto en intención de cumplir con las instrucciones como en valoración y memorización del mensaje (Egan, 2020).

Todos los mensajes mostraban diferentes diseños y formas de lavarse las manos para hacer frente a la COVID-19. Algunos utilizaban infográficos más llamativos, otros se centraban en usar menos texto o en explicar paso a paso como debía de hacerse el lavado de manos correcto. De todos ellos, los que tuvieron mejor recepción los utilizados por las autoridades de Taiwan, Singapur y Reino Unido. Los tres tenían en común que explicaban en sencillos pasos y de forma visual cómo lavarse las manos correctamente, de forma que ampliaban la autonomía de los receptores.

Cuando los gobiernos comunican sobre este tipo de ejercicios, tienen que enfrentar un difícil equilibrio entre proveer de suficiente información a los ciudadanos, pero no demasiadas que sea abrumador para el receptor y pierda el interés. Recientemente en Bangladesh, se presentaron diferentes nudges en estaciones de lavado de manos a las afueras de mezquitas, mercados y estaciones de autobús. Un grupo de investigadores experimentó con los nudges sobre más de 2000 personas y llegaron a la misma conclusión: Ofrecer una guía visual paso a paso aumentaba el lavado de manos (Brown et al, 2020). Sin embargo, añadir demasiados detalles acaba obstaculizando la eficacia del mensaje, ya que el sentimiento de autonomía quedaba eclipsado por otras barreras cognitivas. El mensaje más efectivo conseguía aumentar en 10 puntos porcentuales la probabilidad de que las personas recordaran cómo lavarse las manos adecuadamente (no olvidarse de las palmas y los pulgares, así como hacerlo durante 20 segundos). Pero añadir más pasos al proceso llevaba a que casi unos 5 puntos porcentuales menos recordaran alguno de los detalles mencionados.

Los nudges también pueden ser muy valiosos para promover el lavado de manos en los colegios y universidades. En Filipinas se habían probado una serie de campañas de comunicación que aumentaron la sensibilización sobre el lavado de manos, pero que no consiguieron realmente aumentar su uso efectivo (De Bruck et al., 2017).

Tras varios fracasos, el Departamento de Educación colaboró con UNICEF y otras organizaciones para probar una serie de nudges en la clase que incentivaran a los niños a lavarse las manos. La intervención, que buscaba aumentar la autonomía y relacionalidad de los estudiantes, consistía en dibujar un sendero en el suelo con huellas que llevara a los niños hasta la zona de lavado de manos, donde se pusieron una imagen de ojos observando que los niños se lavaban bien. Además, se incluyó un póster con información sobre cómo lavarse y una flecha señalando el uso del jabón. Estos nudges aumentaron 17 puntos porcentuales el lavado de manos en comparación con el grupo de control tras cuatro meses después de su colocación (Huang et al, 2020). 
GAPP. Nueva Época - N. ${ }^{\circ}$ 25, marzo 2021 - ISSN: 1989-8991 - DOI: https://doi.org/10.24965/gapp.i25.10873 - [Págs. 73-86] Número monográfico - Los nudges y el diseño conductual de políticas públicas

Cómo cambiar comportamientos durante una pandemia: El uso de nudges para enfrentar la COVID-19.

Hugo Cuello Díaz

Este nudge aparentemente simple y muy barato ( 60 \$ por escuela), resultó tener un efecto mucho más grande y significativo que otras medidas y nudges basados en aumentar el sentimiento de competencia que se habían evaluado previamente en colegios.

En otras ocasiones, no solo importa el mensaje y quién lo recibe, sino quien lo envía. Esto se debe a que una norma social es un conjunto de comportamientos individuales que una población entiende como socialmente aceptada por el resto o no. Si el mensaje es recibido por gente de alto prestigio social, puede tener mayor efecto que viniendo de una cuenta oficial ya que aumenta el sentimiento de relacionalidad, como ha ocurrido en otras áreas de comunicación política (Hayes, 2008).

En India, un grupo de investigadores entre los que se encuentra el reciente Premio Nobel de Economía Abhijit Banerjee, evaluaron un nudge presentado por el mismo Banerjee, un personaje muy conocido en India desde que ganó el Nobel. 25 millones de indios recibieron un SMS con un clip de 2.5 minutos, donde Banerjee les pedía personalmente que reportaran si tenían síntomas a las autoridades sanitarias. Diferentes modelos de nudge fueron presentados y comparados con un grupo de control de 3 millones de individuos, que solo recibieron información dirigiéndoles a fuentes de información pública para saber más sobre cómo actuar frente a la pandemia (Banerjee et al., 2020).

El nudge surtió un efecto estadísticamente significativo en los principales indicadores: Dobló el número de registros de síntomas a las autoridades, redujo el movimiento entre diferentes poblaciones casi un $20 \%$ y aumentó el lavado de manos un $7 \%$. Incluso aumentó un $2 \%$ el uso de mascarillas, algo que ni siquiera se mencionaba en ninguno de los nudges.

De esta forma, podemos saber que el rol de los comunicadores tiene un enorme efecto que los diferentes gobiernos deberían considerar si quieren mejorar su comunicación con el cumplimiento de las medidas higiénicas. Además, dada la experiencia previa sobre intervenciones para promocionar el lavado de manos, otro elemento altamente efectivo es intentar convertirlo en un acto habitual.

Los hábitos son acciones que tomamos sin ser plenamente conscientes. Comparar el lavado de manos con un comportamiento habitual y automático puede resultar en un uso más extenso (Aunger et al., 2010). Un nuevo póster del gobierno británico va en esta línea, vinculando el lavado de manos con actividades regulares: Ir al trabajo, sonarse de la nariz y comer ${ }^{4}$.

Esta idea también parece haber funcionado para aumentar el uso de mascarillas. Un experimento reciente en Illinois, Estados Unidos, parece indicar que vincular el uso de mascarillas con medidas de protección existentes y muy extendidas, como ponerse el cinturón o un casco, puede aumentar ampliamente su uso. Ninguno de los otros mensajes y estrategias que probaron las autoridades tuvo un efecto estadísticamente significativo a la hora de persuadir a los americanos a ponerse la mascarilla, aun sabiendo que pueden estar sujetos a multas si no las llevan puestas (Wittenberg, 2020).

\subsection{Nudges para incentivar a la población a quedarse en casa}

Durante los peores momentos de la pandemia, muchos gobiernos instaron a sus ciudadanos a aislarse en casa y no salir si no es por razones de urgencia. Esta medida logró reducir la curva de contagios en los peores momentos de la pandemia, pero también ha tenido muchos efectos negativos, tanto económicos como psicológicos. La evidencia demuestra que los efectos mentales de la cuarentena son extensos, substanciales y pueden mantense en el tiempo, aunque la cuarentena haya acabado (Brooks et al., 2020). Por ello, la forma de comunicar sobre este tipo de medidas necesarias pero muy duras es crucial.

Algunos gobiernos locales en Estados Unidos se dispusieron a probar diferentes nudges que sirvieran para incentivar a la gente a mantener la distancia social y aislarse en casos necesarios. Existen experimentos en más de 10 ciudades de USA y los resultados están empezando a emerger (Bloomberg Cities, 2020). Ciudades como Fayetteville, Kansas City y Newark han usado diferentes pósteres y folletos, así comunicación online y en redes sociales.

En Newark, el mensaje más efectivo basado en el sentimiento de relacionalidad decía: «Los trabajadores que están en primera línea están poniendo su vida en riesgo para mantenerte a salvo. Es tu deber quedarte en casa y ayudar a proteger a otros durante esta crisis». La gente afirmó entender este mensaje de forma más clara que otros, y les ayudó más a recordar las guías de actuación a seguir.

4 El poster se puede consultar en: https://www.britishports.org.uk/system/files/circulars/2020.03.09_a4_poster.pdf. 
GAPP. Nueva Época - N. 25, marzo 2021 - ISSN: 1989-8991 - DOI: https://doi.org/10.24965/gapp.i25.10873 - [Págs. 73-86] Número monográfico - Los nudges y el diseño conductual de políticas públicas

Cómo cambiar comportamientos durante una pandemia: El uso de nudges para enfrentar la COVID-19.

Hugo Cuello Díaz

En Nueva Orleans, se probaron variaciones del mismo SMS pero apoyado en diferentes normas sociales. El mensaje más sencillo "Quédate en casa. Salva vidas», se comparó con otros dos nudges, uno centrado en la responsabilidad y obligación («Es tu deber quedarte en casa»), y el sentimiento de reciprocidad («Enfermeros, doctores y personal sanitario van a trabajar por ti. Quédate en casa por ellos»). De nuevo, el mensaje de reciprocidad resultó ser el más efectivo (Bloomberg Cities, 2020).

En Europa, durante la primera ola la mayoría de gobiernos nacionales y locales usaron mensajes similares para incentivar a la gente a quedarse en casa. Por ejemplo, el Ayuntamiento de Roma experimentó con diferentes nudges en el peor momento de la pandemia y encontró que, entre los jóvenes, el mensaje sobre externalidades («puedes infectar a 45 personas») y normas sociales («la mayoría de italianos piensa») llevó a más italianos a quedarse en casa. Pero estos nudges no influían tanto a otros grupos de población (Schinalia et al., 2020).

En Reino Unido, también están probando varios nudges entre población objetivo. Específicamente, incitando a aquellos que mostraban síntomas a que se aislaran rápidamente, al igual que las personas con vulnerabilidades previas.

El sistema de salud británico (conocido por siglas en inglés, NHS) está experimentando con varios tipos de SMS, justificados en diferentes comportamientos (Burd \& Coleman, 2020). El NHS llegó a enviar en total 7 millones de SMS en una semana con diferentes nudges. Previo al experimento, comprobaron la disposición de los británicos a recibir SMS por parte del NHS, y los resultados fueron esperanzadores: El $85 \%$ estaba de acuerdo en recibir mensajes para estar informados, incluso el $60 \%$ decía estar de acuerdo con recibir uno al día. Usar canales de comunicación alternativos y más directos en momentos de urgencia tiene un impacto fuerte sobre la población y el uso de SMS puede ser una herramienta útil para mejorar la comunicación directa con la población.

De hecho, el experimento ha ofrecido una enorme cantidad de información extra al servicio de salud. En el SMS se pedía a los receptores que respondieran al mensaje informando sobre su situación y si tenían síntomas, para ver cómo estaban evolucionando, ayudando al servicio de salud a prever y organizar cuanta gente ingresaría en las próximas semanas, de forma que se supieran cuantos recursos iban a necesitar. Además, se analizó las respuestas que recibieron de los SMS para identificar cuáles eran las principales preocupaciones sanitarias de los ciudadanos y poder darles respuesta.

Los nudges son una herramienta útil para encomendar a la gente a quedarse en casa en los peores momentos de la pandemia siempre y cuando se cumplan las condiciones de capacidad, oportunidad y motivación. Además, la evidencia demuestra que ofrecer una explicación clara y racional sobre por qué es necesario el aislamiento de una parte de la población puede proteger sobre los efectos psicológicos de la cuarentena, protegiendo la salud mental y el bienestar de aquellos que tienen que mantenerse aislados (Brooks et al., 2020).

Pero los nudges pueden ser útiles tanto durante la época de confinamiento, como a la hora de comunicar cómo se pretende salir de ella. La comunicación gubernamental en esos casos debe tener en cuenta el enorme esfuerzo demandado. Aumentar la confusión y posible extensión de esas medidas podría tener efectos negativos en su cumplimiento.

En Italia, se observó que ir comunicando extensiones de la cuarentena aumenta las probabilidades de que la gente no cumpla con las medidas de confinamiento, ya que produce expectativas que acaban por no cumplirse y crean más confusión. Sin embargo, el mismo estudio demostraba que informar desde el inicio un mayor plazo de cuarentena, aunque luego acabe reduciéndose si las condiciones mejoran, aumentaba la probabilidad de cumplimiento. (Briscese et al., 2020)

\section{PATRONES IDENTIFICADOS Y ESTRATEGIAS DE ÉXITO PARA DISEÑAR NUDGES DURANTE LA COVID-19}

Como se ha visto en los ejemplos mencionados en la sección anterior, el diseño específico e información provista por los nudges tiene un gran efecto en su éxito y cumplimiento, especialmente cuando se desbloquea un motivador cognitivo incentivado por el sentimiento de relacionalidad, autonomía o competencia (Michie et al., 2011). Además, otros elementos como el emisor o el grupo receptor son muy relevantes a la hora de mejorar la eficiencia de la comunicación gubernamental para enfrentar la COVID-19. Algunos patrones a considerar para mejorar la comunicación gubernamental y los nudges son:

Segmentar mensajes por diferentes grupos demográficos: No todos los grupos poblacionales son igualmente propensos a ser influidos por los nudges, ya que el sentimiento de competencia o relacionalidad 
GAPP. Nueva Época - N. ${ }^{\circ}$ 25, marzo 2021 - ISSN: 1989-8991 - DOI: https://doi.org/10.24965/gapp.i25.10873 - [Págs. 73-86] Número monográfico - Los nudges y el diseño conductual de políticas públicas

Cómo cambiar comportamientos durante una pandemia: El uso de nudges para enfrentar la COVID-19.

Hugo Cuello Díaz

puede actuar de forma diferente. Por tanto, en ocasiones es necesario focalizar los esfuerzos de manera efectiva en grupos específicos, por ejemplo, los jóvenes (BIT, 2020).

Las personas jóvenes, especialmente los hombres, tienden a recordar menos contenido de los mensajes gubernamentales y su puntaje de retención es menor. En general, las personas jóvenes pasan menos tiempo leyendo los materiales y comunicaciones oficiales. Además, son los que tienen a declarar una menor conformidad con las instrucciones (Roy-Chowdhury et al., 2020). Por tanto, se necesitan más estudios e información que nos permitan dirigirnos a esos grupos de forma más específica.

Por ejemplo, un estudio centrado en los jóvenes de Estados Unidos encontró que los nudges señalando el riesgo para personas mayores tenía poco efecto en cambiar sus actitudes y comportamientos, por lo que el sentimiento de relacionalidad no surtió efecto en esta dirección (Utych \& Fowler, 2020). Sin embargo, señalar también el riesgo para adultos jóvenes hizo que los jóvenes consideraran el coronavirus como un riesgo mayor.

Reducir la carga cognitiva: La carga cognitiva que las personas pueden atender en situaciones normales es limitada. En momentos de pandemia donde los niveles de ansiedad, frustración e incertidumbre aumentan exponencialmente, la carga cognitiva que podemos soportar se vuelve aún menor y por tanto el sentimiento de autonomía se limita.

Una comunicación gubernamental efectiva que logra la atención de los ciudadanos, es aquella que demanda menor esfuerzo psicológico. Una mala comunicación gubernamental que aumente la incertidumbre, tenga poca claridad y convicción puede tener efectos muy adversos en situaciones de urgencia (Burd \& Coleman, 2020).

Usar comunicación clara y directa: Es crucial atender elementos que favorezcan la limitación de la atención, como reducir el lenguaje complejo y los puntos de fricción. Por tanto, mensajes claros que pongan la acción clave primero, y que sean cortos y directos ayudarán a su fácil comprensión.

En Reino Unido, el mismo mensaje usado para lavarse las manos con 52 palabras tenía peor grado de retención que con 34 palabras (BIT, 2020). A veces, incluso el uso exclusivo de imágenes puede ser más útil, como hicieron en Portland con un póster para ayudar a mantener la distancia social (Bloomberg Cities, 2020).

Evaluar de forma continua: La situación reciente está cambiando de forma dramática en muy poco tiempo. Tanto que a veces es difícil para los ciudadanos seguir todos los cambios, nuevas restricciones y demandas. Esto incrementa la confusión entre la población y puede hacer que algo que funcionaba al principio de la pandemia no funcione más adelante (Everett et al., 2020).

Por ejemplo, un experimento que midió 3 diferentes nudges basados en diferentes comportamientos sociales (enfatizando el beneficio personal, el beneficio social o ambos) tuvo resultados diferentes a lo largo de la evolución de la pandemia: A principios de marzo, el nudge centrado en el beneficio público y social tuvo más impacto que los otros dos. Sin embargo, a finales de abril volvieron a replicar el experimento y se observó que los tres mensajes pasaron a ser igualmente efectivos (Jordan et al., 2020).

Por eso es necesario medir y evaluar de forma continua cuál es la mejor forma de comunicación en cada contexto. Nudges basados en mostrar una víctima identificable de la COVID-19, u otros basados en la sensación de deber y reciprocidad pueden funcionar mejor en ciertos momentos y solo en algunas poblaciones. En otras incluso podría crear el efecto contrario al deseado. Por eso, es clave crear un sistema que permita la evaluación constante.

Contextualizar mensajes al nivel adecuado: Como se ha mencionado, el momento y el lugar es clave para saber que un nudge pueda funcionar, al igual que la dirección del comportamiento que se quiera fortalecer. Pero es importante reconocer la pandemia de la COVID-19 es un evento mundial, que está afectando a todas las partes del mundo con mayor o menos dureza, pero que ha trastocado la estabilidad de todo el planeta.

Por tanto, encuadrar el problema del coronavirus como algo excesivamente local puede no tener los efectos deseados para mejorar el cumplimiento de las medidas de contención del virus, ya que el sentimiento de relacionalidad necesario para el cambio de comportamiento no se orienta en esa dirección. Muchos nudges que se han evaluado intentaron dar una perspectiva local y de comunidad. En Estados Unidos, ciudades como Nueva Orleans quisieron recordar los mensajes de la época del Huracán Katrina para activar a la sociedad; en Kansas se usaron nudges con la imagen de un famoso quarterback local, para aumentar la familiaridad de los ciudadanos. Sin embargo, ninguno de estos mensajes fue realmente efectivo (Bloomberg Cities, 2020).

En general, los mensajes simples o que buscaban despertar el sentido del deber entre la población e incidían en el elemento social fueron más efectivos que los mensajes localistas e individuales. Por tanto, un nudge efectivo tendrá un equilibrio basado en los comportamientos que se quieran promover, que respondan a una motivación concreta pero que no parezcan responsabilidad única de ese grupo poblacional. 
GAPP. Nueva Época - N. ${ }^{\circ}$ 25, marzo 2021 - ISSN: 1989-8991 - DOI: https://doi.org/10.24965/gapp.i25.10873 - [Págs. 73-86] Número monográfico - Los nudges y el diseño conductual de políticas públicas

Cómo cambiar comportamientos durante una pandemia: El uso de nudges para enfrentar la COVID-19.

Hugo Cuello Díaz

Usar mensajes positivos y empáticos: Los nudges que se han desarrollado estos meses parecen indicar que los mensajes sociales y de beneficio público tienen mejor recepción que los únicamente basados es interés individual o en resaltar el elemento de obligatoriedad de cumplimiento. En general, la comunicación positiva y empática aumenta la motivación y ofrece buenos resultados en los lugares donde se ha probado. Especialmente si venían acompañados de cierto grado de flexibilidad y comprensión por parte de las autoridades, como ocurrió en Holanda o en Canadá 5 .

\section{CONCLUSIÓN: IDEAS Y LIMITACIONES EN EL USO DE NUDGES PARA ENFRENTAR LA COVID-19}

El uso de nudges para enfrentar la pandemia de la COVID-19 ofrece un planteamiento complementario al enfoque generalizado, más basado en el uso de herramientas coercitivas. Aquellas zonas y lugares donde los nudges se han usado, se han provisto de evidencia e información útil para mejorar la calidad de la comunicación gubernamental y ha facilitado a los ciudadanos el procesamiento de información urgente y necesaria para el cambio de comportamiento efectivo.

Hay muchos otros terrenos donde los nudges podrían ofrecer aprendizajes útiles y optimizar las estrategias nacionales para la reducción de las medidas restrictivas y de confinamiento. Por ejemplo, promocionando la adopción de apps de rastreo, uso de mascarillas de forma adecuada, o evitar su malgasto y desperdicio para no contaminar el medioambiente. También sería una herramienta enormemente útil para incentivar a los ciudadanos a vacunarse contra la COVID-19 cuando el acceso a las vacunas sea generalizado.

El uso de este enfoque no significa que algunas prohibiciones y restricciones legales no sean necesarias en ciertos casos. Ambos enfoques pueden compenetrarse y utilizarse en los momentos, ocasiones y lugares donde vayan a ser más efectivos. Los nudges pueden ser particularmente efectivos para incentivar comportamientos, especialmente cuando no estamos hablando de un momento puntual de urgencia, sino de un comportamiento que se tendrá que desarrollar durante meses (Hummel \& Maedche, 2019). Pero para momentos excepcionales de colapso sanitario, tendrán que venir acompañados con medidas más restrictivas ya que, por sí solos, es poco probable que vayan a ser suficientemente eficaces.

El enfoque usado por los nudges también cuenta con limitaciones añadidas. Algunos expertos en el diseño de nudges fueron presa de sus propios sesgos y al comienzo de la pandemia minimizaron enormemente su peligro ${ }^{6}$. Incluso en países donde este enfoque se ha utilizado de manera más generalizada, como Reino Unido, los nudges han servido como cabeza de turco para justificar errores políticos que nada tenían que ver con la estrategia basada en la ciencia del comportamiento (The Guardian, 2020). Por tanto, el uso de este enfoque puede causar problemas y servir como justificación para que los responsables políticos no tomen las decisiones necesarias en cada momento.

En definitiva, el uso de nudges durante una pandemia puede ofrecer grandes ventajas si se utiliza de forma inteligente y moderada. Centrada específicamente donde la evidencia empírica demuestre que tiene mayor efectividad. En esos casos, podría servir de alivio para los ciudadanos en algunos terrenos, de forma que no sientan el peso de las prohibiciones constantes y disfruten de flexibilidad y libertad el mayor tiempo posible, aún en tiempos de pandemia mundial.

\section{REFERENCIAS BIBLIOGRÁFICAS}

Abendroth-Dias, K. (27 de abril de 2020). Can behavioural science prepare us for the next pandemic? Apolitical. Consultado el 14 de junio de 2020. https://apolitical.co/en/solution_article/can-behavioural-science-prepare-us-forthe-next-pandemic

Álamo, S. (5 de agosto de 2020). La Policía se abre paso entre los vecinos de Tunte y consigue alojar a los migrantes en el centro previsto. Eldiario.es. Consultado el 13 de agosto de 2020. https://www.eldiario.es/canariasahora/365dias-de-migraciones/vecinos-mantienen-barricada-tension-tunte-pese-59-migrantes-trasladados-dieron-negativocoronavirus_1_6149862.html

\footnotetext{
5 Algunos ejemplos se pueden encontrar en Sunstein, C. R. (2020): "How to Make Coronavirus Restrictions Easier to Swallow". URL: https://www.bloombergquint.com/gadfly/coronavirus-masks-and-social-distancing-can-be-made-more-fun.

6 Véase como ejemplo Sunstein, C. R. (2020): "The Cognitive Bias That Makes Us Panic About Coronavirus" en Bloomberg News Wire. Publicado el 28 febrero 2020. URL: https://www.bloombergquint.com/gadfly/coronavirus-panic-caused-by-probability-neglect.
} 
GAPP. Nueva Época - N. ${ }^{\circ}$ 25, marzo 2021 - ISSN: 1989-8991 - DOI: https://doi.org/10.24965/gapp.i25.10873 - [Págs. 73-86] Número monográfico - Los nudges y el diseño conductual de políticas públicas

Cómo cambiar comportamientos durante una pandemia: El uso de nudges para enfrentar la COVID-19.

Hugo Cuello Díaz

Aunger, R., Schmidt, W.-P., Ranpura, A., Coombes, Y., Mukiri Maina, P., Nkatha Matiko, C. y Curtisa, V. (2010). Three kinds of psychological determinants for hand-washing behaviour in Kenya. Social Science \& Medicine, 70(3), 383391. https://doi.org/10.1016/j.socscimed.2009.10.038

Banerjee, A., Alsan, M., Breza, E., Chandrasekhar, A. G., Chowdhury, A., Duflo, E., Goldsmith-Pinkham, P. y Olken, B. A. (2020). Messages on COVID-19 Prevention in India Increased Symptoms Reporting and Adherence to Preventive Behaviors Among 25 Million Recipients with Similar Effects on Non-recipient Members of Their Communities (NBER Working Paper Series, 27.496). National Bureau of Economic Research. https://doi.org/10.3386/w27496

BIT (2020). Crafting effective communications during a crisis [Webinar: Applying behavioural insights to COVID-19 comms]. Behavioural Insights Team. https://www.bi.team/our-work/webinars/bit-webinar/

Bloomberg Cities (28 de enero de 2020). Messaging that hits home: 5 tips from tests underway in US cities. Consultado el 4 de julio de 2020. https://medium.com/@BloombergCities/messaging-that-hits-home-5-tips-from-testsunderway-in-u-s-cities-9e4f37b4ba63

Bohórquez, L. (10 de julio de 2020). Multas de hasta 3.000 euros por participar en fiestas sin mascarilla. Cadena SER. Consultado el 6 de agosto de 2020. https://cadenaser.com/emisora/2020/07/10/radio_ mallorca/1594380739_413306.html

Briscese, G., Lacetera, N., Macis, M. y Tonin, M. (2020). Compliance with Covid-19 Social-Distancing Measures in Italy: The Role of Expectations and Duration (NBER Working Paper Series, 26.916). National Bureau of Economic Research. http://doi.org/10.3386/w26916

Brooks, S., Webster, R., Smith, L., Woodland, L., Wessely, S., Greenberg, N. y Rubin, G. J. (2020). The psychological impact of quarantine and how to reduce it: rapid review of the evidence. The Lancet, Rapid Review, 395(10.227), 912-920. https://doi.org/10.1016/S0140-6736(20)30460-8

Brown, D., Perera, D. y Kettle, S. (7 de agosto de 2020). COVID-19 prevention: Too much information? The Behavioural Insights Team. Consultado el 9 de agosto de 2020. https://www.bi.team/blogs/covid-19-prevention-too-muchinformation/

Burd, H. y Coleman, C. (8 de abril de 2020). Using behavioural insights to create a Covid-19 text service for the NHS. En The Behavioural Insights Team. Consultado el 4 de junio de 2020. https://medium.com/behavioural-insights/ using-behavioural-insights-to-create-a-covid-19-text-service-for-the-nhs-e062d4071187

Dai, H., Milkman, K. L., Hofmann, D. A. y Staats, B. R. (2015). The impact of time at work and time off from work on rule compliance: the case of hand hygiene in health care. Journal of Applied Psychology, 100(3), 846-862. http://doi. org/10.1037/a0038067

De Buck, E., Van Remoortel, H., Hannes, K., Govender, T., Naidoo, S., Avau, B., Vande Veegaete, A., Musekiwa, A., Lutje, V., Cargo, M., Mosler, H.-J., Vandekerckhove, P. y Young, T. (2017). Approaches to promote handwashing and sanitation behaviour change in low- and middle-income countries: a mixed method systematic review. Campbell Systematic Reviews, 13(1), 1-447. https://doi.org/10.4073/csr.2017.7

Egan, M., Mottershaw, A., Roy-Chowdhury, V., Tagliaferri, G. y Xu, Y. (23 de marzo de 2020). Bright infographics \& minimal text make handwashing posters most effective - result from an online experiment. The Behavioural Insights Team. Consultado el 06 de junio de 2020. https://www.bi.team/blogs/bright-infographics-and-minimal-textmake-handwashing-posters-most-effective/

Everett, J. A. C., Colombatto, C., Chituc, V., Brady, W. J. y Crockett, M. (20 de marzo de 2020). The effectiveness of moral messages on public health behavioral intentions during the COVID-19 pandemic [Preprint]. PsyArXiv. https:// doi.org/10.31234/osf.io/9yqs8

Ford, T. N., Reber, S. y Reeves, R. V. (16 de junio de 2020). Race gaps in COVID-19 deaths are even bigger than they appear. Brookings. Consultado el 20 de agosto de 2020. https://www.brookings.edu/blog/up-front/2020/06/16/racegaps-in-covid-19-deaths-are-even-bigger-than-they-appear/

Gigerenzer, G. y Gaissmaier, W. (2011). Heuristic Decision Making. Annual Review of Psychology, 62, 451-482. https:// doi.org/10.1146/annurev-psych-120709-145346

Google Community Mobility Report (n. d.). Google. Consultado el 6 de julio de 2020. https://www.google.com/covid19/ mobility/

Güell, O. (23 de agosto de 2020). El virus golpea a España por segunda vez. El País. Consultado el 24 de agosto de 2020. https://elpais.com/sociedad/2020-08-22/expertos-coronavirus.html

Hasenbush, A., Miyashita, A. y Wilson, B. (2015). HIV Criminalization in California. Penal Implications for People Living with HIV/AIDS. The Williams Institute. https://www.hivlawandpolicy.org/sites/default/files/HIV\%20 Criminalization\%20in\%20CA\%202015.pdf

Halerm, D. y Harper, H. (21 de mayo de 2020). Behavioural Insights, the WHO and COVID-19. The Behavioural Insights Team. Consultado el 24 de mayo de 2020. https://www.bi.team/blogs/behavioural-insights-the-who-and-covid-19/

Hayes, D. (2008). Does the Messenger Matter? Candidate-Media Agenda Convergence and Its Effects on Voter Issue Salience. Political Research Quarterly, 61(1), 134-146. https://doi.org/10.1177/1065912907306472

Hale, T., Angrist, N., Boby, T., Cameron-Blake, E., Hallas, L., Kira, B., Majumdar, S., Petherick, A., Phillips, T., Tatlow, H. y Webster, S. (10 de diciembre de 2020). Variation in government responses to COVID-19 (Blavatnik School of Government Working Paper, BSG-WP-2020/032). Consultado el 13 de julio de 2020. https://www.bsg.ox.ac.uk/ research/publications/variation-government-responses-covid-19 
GAPP. Nueva Época - N. ${ }^{\circ}$ 25, marzo 2021 - ISSN: 1989-8991 - DOI: https://doi.org/10.24965/gapp.i25.10873 - [Págs. 73-86] Número monográfico - Los nudges y el diseño conductual de políticas públicas

Cómo cambiar comportamientos durante una pandemia: El uso de nudges para enfrentar la COVID-19.

Hugo Cuello Díaz

Huang, C., Le, N., Battle, M. (21 de mayo de 2020). A nudge toward hand hygiene: simple design features improved handwashing among Filipino students [IDinsight Blog]. Medium. Consultado el 06 de junio de 2020. https:// medium.com/idinsight-blog/a-nudge-toward-hand-hygiene-simple-design-features-improved-handwashing-amongfilipino-students-ae4fab1c94db

Hummel, D. y Maedche, A. (2019). How effective is nudging? A quantitative review on the effect sizes and limits of empirical nudging studies. Journal of Behavioral and Experimental Economics, 80, 47-58. https://doi.org/10.1016/j. socec.2019.03.005

Jordan, J., Yoeli, E., Rand, D. (2020). Don't get it or don't spread it? Comparing self-interested versus prosocialmotivations for COVID-19 prevention behaviors [Preprint]. PsyArXiv. https://doi.org/10.31234/osf.io/yuq7x

Judah, G., Donachie, P., Cobb, E., Schmidt, W., Holland, M. y Curtis, V. (2010). Dirty hands: Bacteria of faecal origin on commuters' hands. Epidemiology and Infection, 138(3), 409-414. https://doi.org/10.1017/S0950268809990641

Judah, G., Aunger, R., Schmidt, W.-P., Michie, S., Granger, S. y Curtis, V. (2009). Experimental Pretesting of HandWashing Interventions in a Natural Setting. American Journal of Public Health (suplemento 2, 2009), 99(S2). https://doi.org/10.2105/AJPH.2009.164160

Marcus, J. (2020). The Fun Police Should Stand Down. The Atlantic. Consultado el 05 de agosto de 2020. https://www. theatlantic.com/ideas/archive/2020/08/containing-the-pandemic-isnt-a-job-for-cops/615298/

Martela, F. y Riekki, T. (2018). Autonomy, Competence, Relatedness, and Beneficence: A Multicultural Comparison of the Four Pathways to Meaningful Work. Frontiers in Psychology, 9 (artículo 1.157). https://doi.org/10.3389/ fpsyg.2018.01157

Manun'Ebo, M., Cousens, S., Haggerty, P., Kalengaie, M., Ashworth, A. y Kirkwood, B. (1997). Measuring hygiene practices: a comparison of questionnaires with direct observations in rural Zaïre. Tropical Medicine \& International Health, 2(11), 1.015-1.021. https://doi.org/10.1046/j.1365-3156.1997.d01-180.x

Michie, S., van Stralen, M. M. y West, R. (2011). The behaviour change wheel: A new method for characterising and designing behaviour change interventions. Implementation Science, 6(42). https://doi.org/10.1186/1748-5908-6-42

Ministerio de Sanidad (2020). Estudio ENE-COVID: Informe final estudio nacional de sero-epidemiología de la infección por SARS-COV-2 en España. Ministerio de Sanidad, Gobierno de España. Consultado el 02 de julio de 2020. https://www.mscbs.gob.es/ciudadanos/ene-covid/docs/ESTUDIO_ENE-COVID19_INFORME_ FINAL.pdf

Obliko, N. (16 de mayo de 2020). Behind North America's Lowest Death Rate: A Doctor Who Fought Ebola. Bloomberg (Prognosis). Consultado el 13 de agosto de 2020. https://www.bloomberg.com/news/articles/2020-05-16/a-virusepicenter-that-wasn-t-how-one-region-stemmed-the-deaths

OMS (2012). Communication for behavioural impact (COMBI). A toolkit for behavioural and social communication in outbreak response (reference number WHO/HSE/GCR/2012.13). Consultado el 04 de julio de 2020. https://www. who.int/ihr/publications/combi_toolkit_outbreaks/en/

OMS (6 de abril de 2020a). New WHO/Europe tool for behavioural insights: critical to inform COVID-19 response [WHO Press Release]. Consultado el 11 de julio de 2020. https://www.euro.who.int/en/health-topics/health-emergencies/ pages/news/news/2020/04/new-whoeurope-tool-for-behavioural-insights-critical-to-inform-covid-19-response

OMS (14 de mayo de 2020b). Behavioural insights are valuable to inform the planning of appropriate pandemic response measures [Declaraciones a la prensa del Director Regional para Europa de la OMS, Dr. Hans Henri P. Kluge]. Consultado el 11 de julio de 2020. https://www.euro.who.int/en/media-centre/sections/statements/2020/ statement-behavioural-insights-are-valuable-to-inform-the-planning-of-appropriate-pandemic-response-measures

Pickering, A. J., Davis, J., Blum, A. G., Scalmanini, J., Oyier, B., Okoth, G., Breiman, R. F. y Ram, P. K. (2013). Access to Waterless Hand Sanitizer Improves Student Hand Hygiene Behavior in Primary Schools in Nairobi, Kenya. The American Society of Tropical Medicine and Hygiene, 89(3), 411-418. https://doi.org/10.4269/ajtmh.13-0008

Porter, C. (5 de junio de 2020). The Top Doctor Who Aced the Coronavirus Test. The New York Times. Consultado el 13 de agosto de 2020. https://www.nytimes.com/2020/06/05/world/canada/bonnie-henry-british-columbiacoronavirus.html

Romero, J. M. (26 de julio de 2020). Los muertos de la pandemia en España: 44.868. El País. Consultado el 20 de agosto de 2020. https://elpais.com/sociedad/2020-07-25/las-44868-muertes-de-la-pandemia-en-espana.html

Roy-Chowdhury, V., Perera, D., Tagliaferri, G., Mottershaw, A. y Egan, M. (31 de marzo de 2020). Young men are hardest to engage on coronavirus guidance [Blog]. The Behavioural Insights Team. Consultado el 16 de junio de 2020. https://www.bi.team/blogs/young-men-are-hardest-to-engage-on-coronavirus-guidance/

RTVE.es (18 de agosto de 2020). España, el país europeo con más casos de COVID-19 por población en los últimos 14 días. RTVE. Consultado el 25 de agosto de 2020. https://www.rtve.es/noticias/20200818/espana-pais-europeomas-casos-covid19-poblacion-ultimos-14-dias/2040438.shtml

Ryan, R. M. y Deci, E. L. (2017). Self-Determination Theory: Basic Psychological Needs in Motivation, Development, and Wellnes. The Guilford Press.

Schinalia et al. (2020). Informe no publicado, borrador de resultados del estudio. Recuperado de https://drive.google. com/file/d/1p_u3rHx2LqLeOM49hvDngl-RrWe0SNKB/view?usp=sharing

Sheeran, P. y Web̄b, T. L. (2016). The Intention-Behavior Gap. Social \& Personality Psychology Compass, 10(9), 503518. https://doi.org/10.1111/spc3.12265 
GAPP. Nueva Época - N. ${ }^{\circ}$ 25, marzo 2021 - ISSN: 1989-8991 - DOI: https://doi.org/10.24965/gapp.i25.10873 - [Págs. 73-86] Número monográfico - Los nudges y el diseño conductual de políticas públicas

Cómo cambiar comportamientos durante una pandemia: El uso de nudges para enfrentar la COVID-19.

Hugo Cuello Díaz

Smith, M. (27 de julio de 2020). Face mask use surges after becoming compulsory in shops. YouGov (Health, medicine, \& beauty). Consultado el 02 de agosto de 2020. https://yougov.co.uk/topics/health/articles-reports/2020/07/27/ face-mask-use-surges-after-becoming-compulsory-sho

Southall, A. (7 de mayo de 2020). Scrutiny of Social-Distance Policing as 35 of 40 Arrested Are Black. The New York Times. Consultado el 20 de agosto de 2020. https://www.nytimes.com/2020/05/07/nyregion/nypd-social-distancingrace-coronavirus.html

Thaler, R. H. y Sunstein, C. R. (2009). Nudge: Improving Decisions About Health, Wealth, and Happiness. Yale University Press.

The Guardian (29 de abril de 2020). Revealed: the inside story of the UK's Covid-19 crisis. The Guardian (Covid-19 investigations). Consultado el 17 de junio de 2020. https://www.theguardian.com/world/2020/apr/29/revealed-theinside-story-of-uk-covid-19-coronavirus-crisis

The Lancet HIV (2018). HIV criminalisation is bad policy based on bad science. The Lancet HIV, 5(9) (E473). Consultado el 04 de agosto de 2020. https://doi.org/10.1016/S2352-3018(18)30219-4

Umberson, D. y Montez, J. K. (2010). Social Relationships and Health: A Flashpoint for Health Policy. Journal of Health and Social Behavior, 51 (suplemento 1), S54-S66. https://doi.org/10.1177/0022146510383501

Utych, S. y Fowler, L. (2020). Age-based messaging strategies for communication about COVID-19. Journal of Behavioral Public Administration, 3(1). https://doi.org/10.30636/jbpa.31.151

Umscheid, C. A., Mitchell, M. D., Doshi, J. A., Agarwal, R., Williams, K. y Brennan, P. J. (2015). Estimating the proportion of healthcare-associated infections that are reasonably preventable and the related mortality and costs. Infection Control \& Hospital Epidemiology, 32(2), 101-114. https://doi.org/10.1086/657912

Vindigni, S. M., Riley, P. L. y Jhung, M. (2011). Systematic review: handwashing behaviour in low-to middle-income countries: outcome measures and behaviour maintenance. European Journal of Tropical Medicine \& International Health, 16(4), 466-477. https://doi.org/10.1111/j.1365-3156.2010.02720.x

Wang, Ch., Liu, L., Hao, X., Guo, H., Wang, Q., Huang, J., He, N., Yu, H., Lin, X., Pan, A., Wei, S., Wu, T. (2020). Evolving Epidemiology and Impact of Non-pharmaceutical Interventions on the Outbreak of Coronavirus Disease 2019 in Wuhan, China. MedRxiv. https://doi.org/10.1101/2020.03.03.20030593

West, R. y Michie, S. (2020). A brief introduction to the COM-B Mode of behaviour and the PRIME Theory of motivation. Qeios (ID: WW04E6.2). http://doi.org/10.32388/WW04E6.2

Wittenberg, A. (17 de agosto de 2020). To Get People to Wear Masks, Try Comparing Them to Seatbelts and Helmets. Bloomberg [Citylab]. Consultado el 25 de agosto de 2020. https://www.bloomberg.com/news/articles/2020-08-17/ what-works-to-persuade-people-to-wear-masks 